\title{
Association of genetic variations of genes encoding thrombospondin, type 1, domain-containing 4 and 7A with low bone mineral density in Japanese women with osteoporosis
}

\author{
Seijiro Mori · Ikuyo Kou • Hidenori Sato • \\ Mitsuru Emi · Hideki Ito · Takayuki Hosoi · \\ Shiro Ikegawa
}

Received: 24 March 2008/Accepted: 25 April 2008/Published online: 17 May 2008

(C) The Japan Society of Human Genetics and Springer 2008

\begin{abstract}
Twins and family studies have shown that genetic factors are important determinants of bone mass. Important aspects of bone mineral density (BMD) regulation are endocrine systems, notably hormonal regulation of adrenal corticoids, as indicated by clinical knowledge of glucocorticoid-induced osteoporosis. Glucocorticoid is known to negatively regulate bone mass in vivo, and glucocorticoid increases thrombospondin messenger ribonucleic acid (mRNA) levels. We studied single nucleotide polymorphisms (SNPs) in genes encoding thrombospondin, type 1 , domain-containing 4 and $7 \mathrm{~A}$ (THSD4 and THSD7A) for possible association with lumbar and femoral BMD among 337 Japanese women with osteoporosis who participated in the BioBank Japan project. Genetic variations of THSD4 and THSD7A loci displayed significant association with lumbar and femoral BMD. Most significant correlation was observed for THSD7A SNP rs12673692 with lumbar BMD
\end{abstract}

S. Mori $(\bowtie) \cdot$ H. Sato $\cdot$ H. Ito $\cdot$ T. Hosoi

Department of Internal Medicine,

Tokyo Metropolitan Geriatric Hospital,

35-2 Sakae-cho, Itabashi-ku,

Tokyo 173-0015, Japan

e-mail: mori_seijiro@tmgh.metro.tokyo.jp

I. Kou $\cdot$ S. Ikegawa

RIKEN SNP Research Center, Tokyo, Japan

H. Sato · M. Emi

HuBit Genomix Research Institute, Tokyo, Japan

H. Sato - M. Emi

DNA Chip Research Institute, Yokohama, Japan

T. Hosoi

National Center for Geriatrics and Gerontology,

Aichi, Japan
$(P=0.00017)$. Homozygous carriers of the major $(\mathrm{G})$ allele had the highest BMD $\left[0.886 \pm 0.011 \mathrm{~g} / \mathrm{cm}^{2}\right.$, mean \pm standard deviation (SD)], whereas heterozygous carriers were intermediate $\left(0.872 \pm 0.013 \mathrm{~g} / \mathrm{cm}^{2}\right)$ and homozygous A-allele carriers had the lowest $\left(0.753 \pm 0.023 \mathrm{~g} / \mathrm{cm}^{2}\right)$. THSD4 SNP rs10851839 also displayed strong association with lumbar BMD $(P=0.0092)$. In addition, both THSD7A and THSD4 displayed significant association with femoral BMD in a recessive model $(P=0.036$ and $P=0.0046$, respectively). Results suggest that variations of THSD7A and THSD4 loci may be important determinants of osteoporosis in Japanese women.

Keywords Association study - Bone mineral density . Single nucleotide polymorphism · THSD4 $\cdot$ THSD7A

\section{Introduction}

Osteoporosis, one of the most prevalent disease conditions in the elderly, is defined as a skeletal disorder characterized by compromised bone strength predisposing a person to an increased risk of fracture (National Institutes of Health Consensus Development Panel on Osteoporosis Prevention, Diagnosis, and Therapy 2001). The disease is diagnosed by low bone mineral density (BMD) and the presence of fragility fractures (Orimo et al. 2001). As with many other common diseases, in this disease, multiple factors including genetic variations determine predisposition for the onset or progression of osteoporosis, as indicated by genetic-epidemiological studies (Peacock et al. 2002; Albagha and Ralston 2003). Numerous studies on genetic risks for osteoporosis have been investigated to date, mainly by association studies 
and linkage analysis for BMD as a quantitative trait (Liu et al. 2003).

One important aspect of BMD regulation is the responses of immune systems to changes in sex-steroid environments during aging, which consequently modulate the cytokine production involved in osteoclastogenesis and its activation (Rodan and Martin 2000; Manolagas 1995). In addition to the sex steroids, attention must be paid to adrenal corticoids, as indicated by clinical knowledge of glucocorticoid-induced osteoporosis (Patschan et al. 2001). In fact, glucocorticoid treatment is associated with decreased bone formation and increased risk for fracture (reviewed in Berris et al. 2007). The importance of glucocorticoid in bone metabolism has been implicated not only in the pharmacological situation with glucocorticoid treatment but also in the physiological condition (van Rossum and Lamberts 2004).

In an investigation of glucocorticoid-induced osteoporosis by microarray-based gene expression analysis, it was indicated that glucocorticoid increased thrombospondin messenger ribonucleic acid (mRNA) levels, and that thrombospondins negatively regulate bone mass in vivo, disclosing thrombospondins as novel gene targets, whose regulation by glucocorticoids in osteoblasts may shed light on osteoporosis (Leclerc et al. 2004). A domain called type 1 repeats in thrombospondin- 1 has been shown to bind and activate transforming growth factor-beta (TGF-beta), a cytokine involved in a variety of cellular processes including bone matrix formation (Young and MurphyUllrich 2004). The finding led us to undertake a genetic association study between single nucleotide polymorphisms (SNPs) on thrombospondin, type 1, domaincontaining 4 and 7A (THSD4 and THSD7A) genes and lumbar as well as femoral BMD among 337 Japanese women with osteoporosis who participated in the BioBank Japan project.

\section{Materials and methods}

\section{Subjects}

The osteoporosis case subjects were recruited from the BioBank Japan project according to the Japanese diagnostic criteria for primary osteoporosis (Orimo et al. 2001). We selected 337 osteoporosis subjects that had measurements of both lumbar spine and femoral neck BMD. No participant had medical complications or was undergoing treatment for conditions known to affect bone and lipid metabolism, such as pituitary diseases, hyperthyroidism, primary hyperparathyroidism, liver disease, renal failure, adrenal disease, or rheumatic diseases, and none was receiving estrogen replacement therapy. DNA samples were obtained from peripheral blood and extracted using a standard procedure. We obtained written informed consent from all study subjects, and procedures were approved by the ethics committees of the Institute of Medical Science of the University of Tokyo.

\section{BMD measurement}

Lumbar spine BMD (anteroposterior; L2-L4) and femoral neck BMD were measured by dual-energy X-ray absorptiometry (DXA; Lunar DPX-L, Madison, Lunar, WI, USA) and expressed in grams per centimeter squared $\left(\mathrm{g} / \mathrm{cm}^{2}\right)$. The precision of DPX-L was $0.7 \%$ in coefficient of variation. To calculate adjusted BMD, the measured BMD was normalized for differences in age and body mass index (BMI) by multiple regression analysis using the Instat 3 software package (GraphPad Software, San Diego, CA, USA). The adjustment equation for the study samples was as follows: \{adjusted-BMD $\left.\left(\mathrm{g} / \mathrm{cm}^{2}\right)\right\}=\left\{\right.$ measured-BMD $\left.\left(\mathrm{g} / \mathrm{cm}^{2}\right)\right\}$ $-0.006375 \times[58.39-\{$ age $\quad($ years $)\}]+0.008961 \times$ $\left[23.65-\left\{\right.\right.$ BMI $\left.\left.\left(\mathrm{kg} / \mathrm{m}^{2}\right)\right\}\right]$.

\section{Genotyping procedure}

Genotypes of SNPs rs10851839 (in THSD4) and rs12673692 (in THSD7A) were determined using Invader assay (Mein et al. 2000) according to the manufacturer's instructions. Details of each SNP were archived in the dbSNP at http://www.ncbi.nlm.nih.gov/SNP. SNPs in the THSD4 and THSD7A genes with low minor allele frequency $(<10 \%)$ in Japanese in the HapMap database were excluded from the analysis for statistical power calculation.

\section{Statistical analysis}

Quantitative association between genotypes and adjusted BMD values $\left(\mathrm{g} / \mathrm{cm}^{2}\right)$ was analyzed via one-way analysis of variance (ANOVA) with regression analysis as post hoc test. Three genotypic categories of each SNP were converted into incremental values 0,1 , and 2 corresponding to the number of chromosomes possessing minor allele nucleotides. Significant association was defined when the given $P$ value of the ANOVA $F$ test was $<5 \%(P<0.05)$ (Ota et al. 2001). To ascertain the Hardy-Weinberg equilibrium among genotypes of the subjects, the chi-square test was used $(P>0.05)$.

\section{Results}

Mean values and standard deviations (SD) of the 337 Japanese osteoporosis women were $72.7 \pm 7.3$ years for 
age, $\quad 0.869 \pm 0.157 \mathrm{~g} / \mathrm{cm}^{2}$ for lumbar BMD, and $0.743 \pm 0.081 \mathrm{~g} / \mathrm{cm}^{2}$ for femoral BMD.

We focused on genetic variations in THSD4 and THSD7A genes. SNPs with moderate minor allele frequencies $(>10 \%)$ in Japanese population, rs10851839 (in THSD4) and rs12673692 (in THSD7A), were genotyped among 337 Japanese osteoporosis women who had measurements of both lumbar and femoral BMD in the BioBank Japan project. When the subjects were genotypically categorized into three groups for each SNP, no deviation of genotype frequencies from Hardy-Weinberg equilibrium was detected in the measured SNPs.

ANOVA with linear regression analysis detected a significant correlation between the genotypes of THSD7A SNP, rs12673692, with lumbar BMD $(P=0.00017)$; homozygous carriers of the major $(\mathrm{G})$ allele $(n=162)$ had the highest BMD $\left(0.886 \pm 0.011 \mathrm{~g} / \mathrm{cm}^{2}\right.$, mean $\left.\pm \mathrm{SD}\right)$, whereas heterozygous carriers $(n=144)$ were intermediate $\left(0.872 \pm 0.013 \mathrm{~g} / \mathrm{cm}^{2}\right)$, and homozygous A-allele carriers $(n=27)$ had the lowest BMD $\left(0.753 \pm 0.023 \mathrm{~g} / \mathrm{cm}^{2}\right)$ (Fig. 1a). When the subjects were separated into two groups, the A homozygote and those who bore at least one $\mathrm{G}$ allele $(\mathrm{A} / \mathrm{G}$ and $\mathrm{G} / \mathrm{G})$, the former had significantly lower lumbar BMD than the latter (BMD $0.753 \pm 0.023$ vs $0.879 \pm 0.009 \mathrm{~g} / \mathrm{cm}^{2}, P=0.00004$ ) (Fig. 1b).

THSD4 SNP rs10851839 also displayed strong association with lumbar BMD $(P=0.0092)$. In this case, the minor group, i.e., homozygous A-allele carriers $(n=21)$,
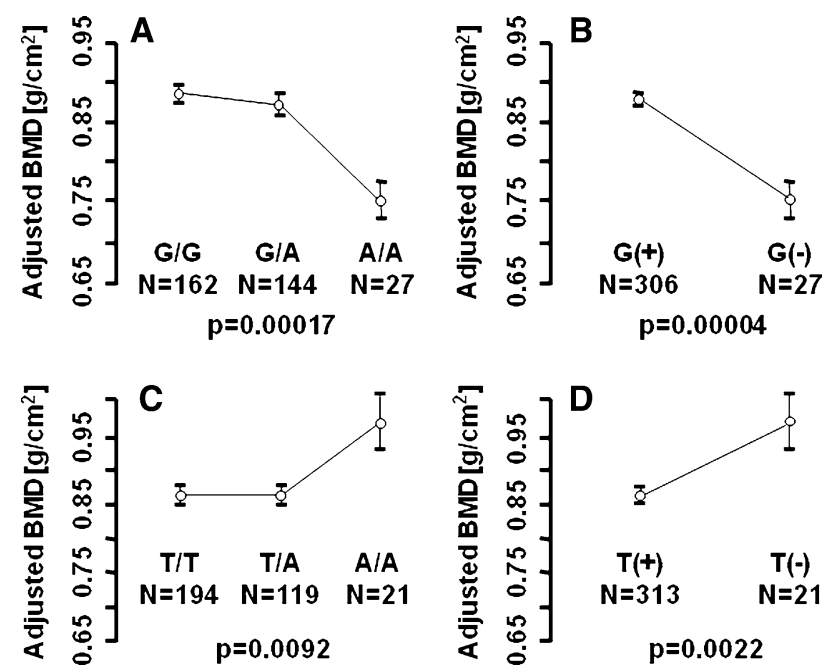

Fig. 1 Lumbar bone mineral density (BMD) among genotypic groups of thrombospondin, type 1, domain-containing 7A (THSD7A) single nucleotide polymorphism (SNP) rs12673692 and THSD4 SNP rs10851839. Values are expressed as means \pm standard deviation. Lumbar BMD among three genotypic group of THSD7A SNP rs12673692 (a). Lumbar BMD in recessive model for THSD7A SNP rs12673692 (b). Lumbar BMD among three genotypic group of THSD4 SNP rs10851839 (c). Lumbar BMD in recessive model for THSD4 SNP rs10851839 (d) had the highest BMD $\left(0.970 \pm 0.040 \mathrm{~g} / \mathrm{cm}^{2}\right)$, heterozygous carriers $(n=119)$ were low $\left(0.862 \pm 0.015 \mathrm{~g} / \mathrm{cm}^{2}\right)$, and homozygous T-allele carriers $(n=194)$ also had low BMD $\left(0.862 \pm 0.015 \mathrm{~g} / \mathrm{cm}^{2}\right)$ (Fig. 1c). When the subjects were separated into two groups, the A homozygote ( $n=21)$ and those who bore at least one T allele (A/T and $\mathrm{T} / \mathrm{T})$, the former had significantly higher lumbar BMD than the latter (BMD $0.970 \pm 0.040$ vs $0.862 \pm 0.009 \mathrm{~g} / \mathrm{cm}^{2}$, $P=0.0022$ ) (Fig. 1d).

For femoral BMD, we analyzed similar relationships between alleles of THSD7A SNP rs12673692 and THSD4 SNP rs10851839 with femoral BMD in the same sample set. Both THSD7A and THSD4 revealed significant association with femoral $\mathrm{BMD}$ in a recessive model. For THSD7A SNP rs12673692 the A homozygote had significantly lower femoral BMD than those who bore at least one $\mathrm{G}$ allele (A/G and $\mathrm{G} / \mathrm{G})$ (BMD $0.712 \pm 0.013$ vs $0.746 \pm 0.005 \mathrm{~g} / \mathrm{cm}^{2}, P=0.036$ ) (Fig. 2a). For THSD4 SNP rs10851839, the A homozygote had significantly higher femoral BMD than those who bore at least one $\mathrm{T}$ allele (A/T and $\mathrm{T} / \mathrm{T}) \quad(\mathrm{BMD} \quad 0.791 \pm 0.015 \quad$ vs $0.740 \pm 0.005$ g/cm ${ }^{2}, P=0.0046$ ) (Fig. 2b).

These results suggest that genetic variations of THSD7A and THSD4 loci are related to development of osteoporosis. We hypothesized that A-allele homozygote for THSD7A SNP rs12673692 is an important risk factor for osteoporosis, whereas A-allele homozygote for THSD4 SNP rs10851839 is a protective factor for osteoporosis in adult Japanese women.

\section{Discussion}

For the first time, this study detected significant association between genotypes of SNPs on THSD4 and THSD7A genes with adjusted levels of lumbar and femoral BMD among adult Japanese women osteoporosis patients.

Proteins encoded by the thrombospondin, type 1, domain-containing gene family possess a unique sequence



Fig. 2 Femoral bone mineral density (BMD) among two genotypic groups in recessive model for thrombospondin, type 1, domaincontaining 7A (THSD7A) single nucleotide polymorphism (SNP) rs12673692 (a) and THSD4 SNP rs10851839 (b). Values are expressed as mean \pm standard deviation 
called thrombospondin type 1 repeats. The type 1 repeats in thrombospondin- 1 bind and activate TGF-beta, a cytokine involved in a variety of cellular processes including bone matrix formation (Young and Murphy-Ullrich 2004). TGFbeta, abundant in bone matrix, is known to be an important regulator of osteoblast proliferation and differentiation and has been shown to directly affect bone formation in vivo (Cohen 2003). Therefore, the possibility that the gene family members THSD4 and THSD7A play an important role in the pathogenesis of osteoporosis possibly by modulating the activity of TGF-beta may deserve further investigation. Longitudinal studies in large cohorts, as well as functional studies, may clarify the molecular events by which the THSD7A and THSD4 variations bring about alterations in bone metabolism.

A limitation of this study is the way subjects were recruited. They were all diagnosed with osteoporosis. In addition, the effects of polymorphisms on BMD may differ between the sites of measurements because of the possible difference in age-related changes in BMD between lumbar spine and proximal femur. Further studies are required to extrapolate the results to the general population.

In summary, we showed significant association of the intronic variants in the THSD7A and THSD4 genes with lumbar and femoral neck BMD of an osteoporosis population of adult Japanese women. A possibility cannot be ruled out, however, that this SNP marker may itself be in linkage disequilibrium with other unmeasured and functional variants at or near the THSD genes that are the true mechanistic basis for the associations. Functional studies are required to rule out these possibilities.

Acknowledgments We appreciate all the volunteers and participating institutions of BioBank Japan, University of Tokyo, Institute of Medical Science, Japan, for valuable clinical data and samples. We also thank Prof. Yusuke Nakamura and Dr. Michiaki Kubo in RIKEN SNP Research Center, Tokyo, Japan, for their generous support in our accomplishment of this work. The BioBank Japan Project on the implementation of personalized medicine was supported by the Ministry of Education, Culture, Sports, Science and Technology (MEXT). This work was supported by a grant from BioBank Japan (No 03023120).

\section{References}

Albagha OM, Ralston SH (2003) Genetic determinants of susceptibility to osteoporosis. Endocrinol Metab Clin North Am 32:6581

Berris KK, Repp AL, Kleerekoper M (2007) Glucocorticoid-induced osteoporosis. Curr Opin Endocrinol Diabetes Obes 14:446-450

Cohen MM Jr (2003) TGF-beta/Smad signaling system and its pathologic correlates. Am J Med Genet A 116:1-10

Leclerc N, Luppen CA, Ho VV, Nagpal S, Hacia JG, Smith E, Frenkel B (2004) Gene expression profiling of glucocorticoid-inhibited osteoblasts. J Mol Endcrinol 33:175-193

Liu YZ, Liu YJ, Robert RR, Deng HW (2003) Molecular studies of identification of genes for osteoporosis: the 2002 update. Endocrinology 177:147-196

Manolagas SC (1995) Role of cytokines in bone resorption. Bone 17:63-67

Mein CA, Barratt BJ, Dunn MG, Siegmund T, Smith AN, Esposito L, Nutland S, Stevens HE, Wilson AJ, Phillips MS, Jarvis N, Law S, de Arruda M, Todd JA (2000) Evaluation of single nucleotide polymorphism typing with invader on PCR amplicons and its automation. Genome Res 10:330-343

NIH Consensus Development Panel on Osteoporosis Prevention, Diagnosis, and Therapy (2001) JAMA 285:785-795

Orimo H, Hayashi Y, Fukunaga M, Sone T, Fujiwara M, Shiraki M, Kushida K, Miyamoto S, Soen S, Nishimura J, Oh-hashi Y, Hosoi T, Gorai I, Tanaka H, Igai T, Kishimoto H (2001) Diagnostic criteria for primary osteoporosis: year 2000 revision. J Bone Miner Metab 19:331-337

Ota N, Nakajima T, Nakazawa I, Suzuki T, Hosoi T, Orimo H, Inoue S, Shirai Y, Emi M (2001) A nucleotide variant in the promoter region of the interleukin- 6 gene associated with decreased bone mineral density. J Hum Genet 46:267-272

Patschan D, Loddenkemper K, Buttgereit F (2001) Molecular mechanisms of glucocorticoid-induced osteoporosis. Bone 29:498-505

Peacock M, Turner CH, Econs MJ, Foroud T (2002) Genetics of osteoporosis. Endocr Rev 23:303-326

Rodan GA, Martin TJ (2000) Therapeutic approaches to bone diseases. Science 289:1508-1514

van Rossum EF, Lamberts SW (2004) Polymorphisms in the glucocorticoid receptor gene and their associations with metabolic parameters and body composition. Recent Prog Horm Res 59:333-357

Young GD, Murphy-Ullrich JE (2004) The tryptophan-rich motifs of the thrombospondin type 1 repeats bind VLAL motifs in the latent transforming growth factor-beta complex. J Biol Chem 279:47633-47642 